\title{
Weiterbildungsangebote im Kontext von Migration und Flucht - Ergebnisse und Folgerungen am Beispiel der Volkshochschulen in Nordrhein-Westfalen
}

\author{
Halit Öztürk
}

Eingegangen: 18. Mai 2018 / Angenommen: 29. August 2018 / Online publiziert: 13. September 2018 (C) Der/die Autor(en) 2018

Zusammenfassung Volkshochschulen nehmen ihren Bildungsauftrag flächendeckend, thematisch breit und für alle Bürgerinnen und Bürger wahr. Um gleiche Partizipationsmöglichkeiten auch für alle Bevölkerungsgruppen zu schaffen, stehen sie vor der Herausforderung, ihre Arbeit kontinuierlich an gesellschaftliche Entwicklungen anzupassen. Organisationsumfassende Konzepte wie Diversity Management und Interkulturelle Öffnung bieten einen Rahmen, der verschiedene Ebenen der Weiterbildungsarbeit berücksichtigt und auf ihren Beitrag zur Anerkennung, Wertschätzung und Einbeziehung gesellschaftlicher Vielfalt innerhalb und außerhalb der Organisation hin befragt. Migration und Flucht sind dabei Themen, die in den vergangenen Jahren einen neuen Aufmerksamkeitsschub erfahren haben. Der Beitrag fokussiert die Programm- und Angebotsgestaltung für Erwachsene mit einem sogenannten Migrationshintergrund an Volkshochschulen in Nordrhein-Westfalen. Aus einer 2016 durchgeführten empirischen Bestandsaufnahme über organisationsumfassende Maßnahmen im Kontext von Migration werden ausgewählte Ergebnisse präsentiert und um eine inhaltsanalytische Webseitenanalyse ergänzt. Um die Angebotsgestaltung als integrierten Teil der Weiterbildungsarbeit an Volkshochschulen zu betrachten, werden auch einige Ergebnisse auf den Analyseebenen des Personals und der Organisation vorgestellt. Abschließend werden konkrete Herausforderungen auf allen Analyseebenen benannt.

Schlüsselwörter Migration · Weiterbildung · Volkshochschulen · Organisationsforschung

\footnotetext{
Publisher's Note Springer Nature remains neutral with regard to jurisdictional claims in published maps and institutional affiliations.

Prof. Dr. H. Öztürk $(\bowtie)$

Institut für Erziehungswissenschaft, Arbeitsbereich Erwachsenenbildung/Weiterbildung, Westfälische Wilhelms-Universität Münster, Georgskommende 33, 48143 Münster, Deutschland

E-Mail: halit.oeztuerk@wwu.de
} 


\title{
Course offers in adult education in the context of migration and flight-results and conclusions from the example of adult education centers in North Rhine-Westphalia
}

\begin{abstract}
Adult education centers ("Volkshochschulen") realize their educational mission nationwide, in a wide range of educational topics and for all citizens. In order to foster equal participation opportunities for all population groups, they face the challenge of adapting their work to social developments. Organizational approaches such as diversity management and intercultural opening provide a framework that considers different levels of continuing education work and discusses their contribution to the recognition, appreciation and inclusion of social diversity within and outside the organization. Migration and flight are topics that have experienced a new wave of attention in recent years. The article focuses on the arrangement of programs and course offers for adults with a so-called migration background at adult education centers in North Rhine-Westphalia. From an empirical review of organizational measures in the context of migration carried out in 2016, the article presents selected results, supplemented by a website content analysis. In order to view the arrangement of course offers as an integrated part of continuing education work at adult education centers, some results are also presented at the analysis levels of the staff and the organization. Finally, concrete challenges are identified at all three levels.
\end{abstract}

Keywords Migration · Continuing education · Adult education centres · Organization research

\section{Einführung und Ausgangslage}

In Deutschland hat sich eine Vielfalt von Einrichtungen sehr unterschiedlicher Größe, Struktur und Trägerschaft herausgebildet, die Weiterbildung anbieten. So finden sich neben öffentlichen Weiterbildungseinrichtungen von Bund, Ländern und Kommunen insbesondere Einrichtungen gesellschaftlicher Großgruppen, gemeinnützig tätige Einrichtungen und kommerziell agierende Anbieter sowie Betriebe und Einzeltrainerinnen und -trainer (vgl. Gnahs und Bilger 2013, S. $110 \mathrm{ff}$.). Unter diesen vielfältigen Anbietern stehen die Volkshochschulen (VHS) in Deutschland seit jeher in öffentlicher Verantwortung und haben sich seit ihrer Entstehung Ende des 19. Jahrhunderts in besonderer Weise den Zielen der Bildungsgerechtigkeit, sozialen Partizipation und gesellschaftlichen Integration verschrieben (vgl. Olbrich 2001, S. $148 \mathrm{ff}$.).

Bis heute machen die überwiegend kommunal oder vereinsmäßig organisierten VHS unmissverständlich deutlich, dass sie allen Menschen - unabhängig von Alter, Geschlecht, sozialen oder ökonomischen Voraussetzungen, Behinderung, Weltanschauung und Herkunft - offenstehen (vgl. DVV 2011, S. 4; Süssmuth und Eisfeld 2018, S. 764 f.). Mit den diversen zielgruppenspezifischen Angeboten werden dabei 
auch Menschen mit einem sogenannten „Migrationshintergrund“" gezielt als „,besondere Adressatengruppe“ angesprochen (vgl. Huntemann und Reichart 2017, S. 23).

Bereits ein kurzer Blick auf die historische Entwicklung solcher Angebote an VHS zeigt, dass diese in Deutschland in enger Verbindung mit den jeweils aktuellen Migrationsbewegungen und -politiken stehen. Großstädtische VHS begannen in den 1960er Jahren punktuell, für angeworbene Arbeitsmigrantinnen und -migranten Angebote im Bereich Deutsch, Grundbildung, Beratung und Unterstützung bei Alltagsproblemen und Behördenangelegenheiten zu organisieren (vgl. Ruhlandt 2016, S. 28 f.). ${ }^{2}$ Mit dem Familiennachzug und der dauerhaften Niederlassung vieler zugewanderter Familien in Deutschland stiegen auch die Bemühungen der VHS, in den Bereichen gesellschaftlicher, politischer, beruflicher und kultureller Bildung über Deutsch- und Orientierungskurse hinausgehende Angebote zu machen (ebd., S. $29 \mathrm{ff}$.).

Heute führen nahezu alle VHS in Deutschland regelmäßig Zielgruppenangebote für Erwachsene mit Migrationshintergrund durch und sind dabei im Vergleich zu anderen Weiterbildungsanbietern mit weitem Abstand führend (vgl. Ambos et al. 2017, S. 11 f.; Robak und Petter 2014, S. 35 f.). Im wbmonitor ${ }^{3}$ werden etwa im Bereich der nicht-berufsbezogenen Weiterbildung vorwiegend allgemeine Deutschkurse genannt, gefolgt von Kursen im Bereich Alltagskompetenzen und Alphabetisierung. Im Bereich der berufsbezogenen Weiterbildung dominieren dagegen berufsbezogene Deutschkurse, Orientierungsangebote und Basisqualifizierungen (vgl. Ambos et al. 2017, S. 15 ff.). Auch die Ergebnisse der Volkshochschul-Statistik ${ }^{4}$ bestätigen diese Befunde: Im Berichtsjahr 2016 richteten sich von insgesamt 594.000 Kursen 48.588 Kurse allein an Menschen mit Migrationshintergrund. Die Kursangebote für diese Zielgruppe nehmen in nahezu allen Programmbereichen kontinuierlich zu. Allein zwischen 2014 und 2016 stieg das zielgruppenspezifische Kursangebot der VHS für diese Bevölkerungsgruppe, beeinflusst durch die stark gestiegene Fluchtmigration in

\footnotetext{
1 Unter Personen mit Migrationshintergrund werden in Anlehnung an das Statistische Bundesamt (2017) alle Personen erfasst, die selbst oder deren Eltern seit Bestehen der Bundesrepublik nach Deutschland zugewandert sind. Diese Definition ist Grundlage einer Vielzahl von Studien, die sich mit Migration im Rahmen der Weiterbildung auseinandersetzen (im Überblick siehe Öztürk 2014). Auch den Studienergebnissen, die im Rahmen dieses Artikels berichtet werden, liegt diese Definition zugrunde (vgl. Öztürk und Reiter 2017). Wenngleich die Bezeichnung „Migrationshintergrund“ bereits eine breite Akzeptanz in Wissenschaft und Öffentlichkeit gefunden hat, wird sie insbesondere als konstruierte und machtvolle Differenzierung zunehmend kritisch hinterfragt (vgl. z.B. Eschenbach 2016, S. 48 ff.; Heinemann 2018, S. 16f.).

2 Sowohl das Thema Flucht als auch geflüchtete Menschen sind bereits seit Jahrzehnten präsent in der Erwachsenenbildung mit einer Vielzahl an Weiterbildungs- und Beratungsangeboten (vgl. Falkenstern und Käpplinger 2017, S. 12f.).

3 Eine jährlich bundesweit durchgeführte Anbieterbefragung des Bundesinstituts für Berufsbildung (BIBB) und des Deutschen Instituts für Erwachsenenbildung (DIE) zu Leistungen und Strukturen der allgemeinen und beruflichen Weiterbildung in Deutschland. Für nähere Informationen siehe https:// wbmonitor.bibb.de/.

4 Eine jährlich bundesweit durchgeführte Vollerhebung des Deutschen Volkshochschul-Verbands (DVV) und des DIE unter den 899 Mitgliedseinrichtungen der 16 Volkshochschul-Landesverbände (Berichtsjahr 2016) zu institutionellen Merkmalen und Personal- und Angebotsstrukturen sowie Finanzierung und Veranstaltungsdaten. Für nähere Informationen siehe https://www.die-bonn.de/weiterbildung/statistik/vhsstatistik/.
} 
diesen Jahren, um rund $68 \%$. Die Deutschkurse überwiegen allerdings deutlich, ihr Anteil lag im Jahr 2016 beispielsweise bei rund $97 \%$ (vgl. Huntemann und Reichart 2015, S. 40; 2017, S. 54).

Solche fortwährenden Bemühungen um Weiterbildungsteilhabe - auch durch eine gezielte Zielgruppenansprache - erscheinen insbesondere vor dem Hintergrund der häufigen Forderung nach lebenslangem Lernen (siehe z.B. Europäische Union 2015) bei gleichzeitig tiefer Kluft in der Weiterbildungsbeteiligung zwischen den verschiedenen Bevölkerungsgruppen (siehe z. B. BMBF 2018, S. 13 ff.) nachvollziehbar und notwendig. Dies trifft insbesondere auf zugewanderte Erwachsene $\mathrm{zu}$, deren Weiterbildungsbeteiligung deutlich niedriger ist als die der Menschen ohne Migrationshintergrund, bzw. der in Deutschland geborenen und aufgewachsenen Menschen mit Migrationshintergrund (die sog. zweite Generation) (vgl. BMBF 2018, S. 19 ff.; Öztürk und Reiter 2016, S. 53 ff.). ${ }^{5}$

Vor diesem Hintergrund sind die VHS, wie alle Weiterbildungseinrichtungen, dazu aufgefordert, ihre organisationalen Strukturen und Praktiken dahingehend zu gestalten, dass allen gleiche Zugangs- und Partizipationschancen eröffnet werden (vgl. Öztürk und Reiter 2017, S. 28 ff.). Die empirische Weiterbildungsforschung liefert jedoch noch kein umfassendes Bild über den Umgang von Weiterbildungseinrichtungen mit migrations- und fluchtbedingten gesellschaftlichen Entwicklungen und die damit verbundenen vielschichtigen Herausforderungen (vgl. Öztürk 2014, S. 53 ff.). Dies war Anlass für eine im Frühjahr 2016 durchgeführte empirische Bestandsaufnahme zu organisationsweiten Strategien und Maßnahmen von Weiterbildungseinrichtungen im bevölkerungsreichsten und durch einen vergleichsweise hohen Anteil von Menschen mit Migrationshintergrund geprägten Bundesland Nordrhein-Westfalen (NRW).

Auf Basis der Daten dieses Forschungsprojekts fokussiert der folgende Beitrag exemplarisch die VHS und deren Umgang mit Migration und Flucht auf der Analyseebene der Angebote. ${ }^{6}$ Vor dem Hintergrund, dass ein geplanter Wandel in Weiterbildungseinrichtungen in Richtung gleicher Partizipationschancen nur organisationsumfassend gestaltet werden kann (vgl. Öztürk und Reiter 2017, S. 28; S. 40), werden auch die weiteren Analyseebenen der Organisation und des Personals kurz erörtert.

Dazu werden im Folgenden nach einer knappen Darstellung des konzeptionellen Rahmens dieses Forschungsprojekts, einschließlich der für diesen Beitrag unternommenen Zusätze und Ergänzungen, einige ausgewählte Ergebnisse präsentiert. Abschließend werden die Ergebnisse in einem Fazit zusammengefasst und daraus re-

\footnotetext{
5 Während die Unterrepräsentanz von Erwachsenen der ersten Zuwanderergeneration, also jener Personen, die nach Deutschland zugewandert sind und damit ihre Bildungslaufbahn nicht ausschließlich in Deutschland verbracht haben, in der Weiterbildung hinlänglich bekannt ist (siehe z. B. Öztürk und Kaufmann 2009; Öztürk 2016), stellt die Weiterbildungssituation von anerkannten bzw. geduldeten Geflüchteten und Asylsuchenden ein Desiderat der Weiterbildungsforschung dar. Insofern sollten diese Personengruppen - auch vor dem Hintergrund ihres weiter steigenden Anteils an der Gesamtbevölkerung - in Forschung und Praxis der Weiterbildung mehr Beachtung finden.

${ }^{6}$ Für die bereits veröffentlichten detaillierten Ergebnisse dieses Forschungsprojekts siehe Öztürk und Reiter (2017).
} 
sultierende Handlungsempfehlungen für eine diversitätsorientierte Angebotsplanung und -entwicklung sowie Organisations- und Personalentwicklung in VHS formuliert.

\section{Konzeptioneller Rahmen des Forschungsprojekts}

Zur Beantwortung der Frage nach dem Umgang mit gesellschaftlicher Vielfalt und organisationalem Wandel können unterschiedliche Ansätze herangezogen werden. Den Rahmen dieses Forschungsprojekts bilden dabei die besonders in den letzten zwei Jahrzehnten in Deutschland zunehmend rezipierten und adaptierten Konzepte Diversity Management und Interkulturelle Öffnung (vgl. Öztürk und Reiter 2017, S. 28 ff.; Ruhlandt 2016, S. 48 ff.). Wenngleich diese Konzepte jeweils unterschiedliche Entstehungs- und Begründungszusammenhänge aufweisen, zielen sie doch grundsätzlich - knapp zusammengefasst - auf Anerkennung, Wertschätzung und Einbeziehung gesellschaftlicher Vielfalt innerhalb und außerhalb der Organisation. Zielsetzungen sind in diesem Zusammenhang beispielsweise

- der Abbau von Partizipations- und Zugangsbarrieren bzw. die Eröffnung von gleichen und angemessenen Partizipations- und Zugangsmöglichkeiten,

- die Sensibilisierung aller Organisationsmitglieder für eine äußerst von Diversität geprägte Adressaten- und Teilnehmendenschaft und

- die aktive Förderung eines aufrichtigen und wertschätzenden Umgangs mit vielfältigen biografischen Erlebnissen, physischen, ökonomischen und sozialen Realitäten.

Damit werden kontinuierliche Entwicklungs-, Lern- und Veränderungsprozesse insbesondere auf Organisations-, Personal- und Angebotsebene angestrebt (vertiefend siehe beispielsweise Öztürk und Reiter 2017; Walgenbach 2017; Vanderheiden und Mayer 2014).

Vor diesem Hintergrund wurde das eigene Forschungsprojekt „Diversity im Kontext von Migration in der Weiterbildung" (DiMiWeit) in drei Analyseebenen strukturiert. Untersucht wurden auf der Ebene:

1. Organisation die Relevanzzuschreibung von Migration, die Bekanntheit und Umsetzung von Konzepten wie „Diversity Management“ und „Interkulturelle Öffnung" sowie die damit verbundenen Erwartungshaltungen

2. Personal die Rekrutierung, Beschäftigung und Positionierung von Mitarbeitenden mit Migrationshintergrund

3. Angebote die Zielgruppenangebote einschließlich der Aktivitäten im Bereich der Öffentlichkeitsarbeit und Zielgruppenansprache sowie der Repräsentation von Teilnehmenden mit Migrationshintergrund im Regelangebot (vgl. Öztürk und Reiter 2017, S. 54).

Ausgehend von diesem konzeptionellen Bezugsrahmen wurde eine weitgehend standardisierte Online-Befragung konzipiert und unter Weiterbildungseinrichtungen in NRW durchgeführt. Auf diese Weise konnte auf Basis von Eigenmitteln eine umfangreiche Stichprobe und eine breite Abdeckung verschiedener Organisationsbereiche realisiert werden. Die vertiefende Analyse einzelner Aspekte wurde im 
Rahmen der Bestandsaufnahme jedoch nicht angestrebt. Im Rückgriff auf unterschiedliche Datenbanken wie z. B. das Bildungsportal des Ministeriums für Schule und Weiterbildung des Landes NRW, Portale der Landesarbeitsgemeinschaften sowie Webseiten und Verzeichnisse unterschiedlicher Trägergruppen konnten 675 Weiterbildungseinrichtungen identifiziert und per E-Mail zur Teilnahme an der Befragung eingeladen werden. Teilgenommen daran haben 260 Weiterbildungseinrichtungen (Rücklaufquote 38,5\%), darunter sowohl Einrichtungen unterschiedlicher Trägerschaften mit öffentlichen und gemeinnützigen Interessen als auch Einrichtungen in privater Trägerschaft.

Der Anteil der VHS an allen befragten Einrichtungen beträgt gut ein Viertel $(27,3 \%, N=71$ mit einer Rücklaufquote von 53,8\%) (vgl. Öztürk und Reiter 2017, S. $56 \mathrm{ff}$.). Der weit überwiegende Teil der an der Befragung beteiligten VHS $(63,4 \%)$ befindet sich im städtischen Raum (Einwohnerzahl: 20.001 bis 100.000 Einwohner). $29,6 \%$ der VHS liegen im großstädtischen Raum (100.001 bis 500.000 Einwohner). Lediglich ein kleiner Teil der befragten VHS liegt im kleinstädtischen und ländlichen Raum mit einer Einwohnerzahl von unter 20.000 (4,2\%), bzw. im metropolitanen Raum mit über 500.000 Einwohnerinnen und Einwohnern $(2,8 \%)$.

Diese nicht repräsentativen Daten bilden damit im Folgenden die Basis zur Beantwortung der Fragestellung, wie Volkshochschulen mit gesellschaftlichen Entwicklungen von Migration und Flucht umgehen. Diese Ergebnisse werden durch eine im Rahmen dieses Beitrags ergänzend durchgeführte Webseitenanalyse angereichert. Die Leitfrage dabei lautet, ob Projekte und Initiativen existieren, die den engen zielgruppenspezifischen Rahmen, wie er z. B. in Deutsch- und Integrationskursen gegeben ist, um partizipative bzw. dialogorientierte Elemente erweitern. Gesichtet wurden jeweils zehn VHS-Webseiten in nordrhein-westfälischen kreisfreien Städten oder Kreisen, die den höchsten bzw. niedrigsten Bevölkerungsanteil an Personen mit Migrationshintergrund aufweisen, ${ }^{7}$ um mögliche Unterschiede aufzuzeigen. Die Webinhalte wurden mithilfe der zusammenfassenden qualitativen Inhaltsanalyse ausgewertet (vgl. Mayring 2015). Zudem fließen die auf den Webseiten des Deutschen Volkhochschul-Verbands e. V. (DVV) und des Landesverbands der Volkshochschulen von NRW e. V. verfügbaren schriftlichen Informationen (u.a. Positionspapiere, Presseerklärungen, Jahresberichte) in die Analyse ein.

\section{Volkshochschulen im Umgang mit Migration und Flucht}

Im Folgenden werden die empirischen Ergebnisse der zuvor dargestellten Befragung sowie der ergänzenden Inhaltsanalyse von Webseiten ausgesuchter VHS und ihrem Umgang mit Migration und Flucht vorgestellt. Nach der Analyseebene der Angebote werden ausgewählte Ergebnisse auf Ebene des Personals und der Organisation ergänzend dargestellt. Alle Ergebnisse stützen sich dabei auf die Selbstauskünfte der befragten Einrichtungen, die mithilfe verschiedener Frageformate (Filterfragen, ge-

\footnotetext{
7 Dazu wurde auf die „Integrationsprofile der kreisfreien Städte und Kreise“ des Ministeriums für Arbeit, Integration und Soziales (MAIS 2016) zurückgegriffen.
} 
schlossene Fragen mit Einfach- und Mehrfachnennungen, offene Fragen) erschlossen wurden (vgl. Öztürk und Reiter 2017, S. 54f.).

\subsection{Umgang auf Ebene der Angebote}

Auf dieser Analyseebene ist der Fokus auf Angebote und Aktivitäten der VHS für die Adressatengruppe Erwachsener mit Migrationshintergrund ${ }^{8}$ gerichtet, die ein sichtbarer Ausdruck der vielfältigen Bestrebungen der VHS in diesem Bereich sind. Neben einer Vielzahl von Angeboten rund um die Themen Migration, Diversität, Flucht und Asyl für einen breiten Adressatenkreis organisieren 97,1\% der befragten VHS Kursangebote speziell für Erwachsene mit Migrationshintergrund. Dabei werden Integrationskurse (95,5\%), allgemeine Deutschkurse $(91,0 \%)$ und Kurse zur Grundbildung und Alphabetisierung $(88,1 \%)$ von einem Großteil der befragten VHS angeboten (s. Abb. 1). Im Vergleich zu anderen Kursthemen bilden diese Angebote auch die Mehrheit der zielgruppenspezifischen Angebote, was sich auch mit Ergebnissen der Volkshochschul-Statistik und des wbmonitor deckt (vgl. Ambos et al. 2017, S. 15 ff.; Huntemann und Reichart 2017, S. 54).

Weitere häufig genannte Angebote sind berufsbezogene Deutschkurse (44,8\%), allgemeine Beratungsangebote (43,3\%), Kurse rund um Themen wie Mensch, Gesellschaft und Politik (29,9\%) sowie berufliche Qualifizierungsangebote für den Einstieg $(22,4 \%)$. Im Gegensatz dazu werden Kurse vor allem im Bereich Medien und Management (jeweils 1,5\%) weniger häufig zielgruppenspezifisch angeboten (s. Abb. 1). Unter Sonstiges nannten die befragten VHS u. a. Anerkennungsberatung und Gesprächskreise. Darüber hinaus wurden in der Online-Befragung auch Projekte und Initiativen genannt, die den engen zielgruppenspezifischen Rahmen um partizipative bzw. dialogorientierte Elemente erweitern (wie z. B. interkulturelle Frauencafés, interkulturelle und interreligiöse Gesprächskreise) (vgl. Öztürk und Reiter 2017, S. 96 ff.). ${ }^{9}$

Die Analyse von insgesamt 20 VHS-Webseiten brachte zusammengefasst folgende Ergebnisse:

Partizipative bzw. dialogorientierte Projekte und Initiativen waren ausschließlich an VHS in kreisfreien Städten mit einem hohen Bevölkerungsanteil an Personen mit Migrationshintergrund - vorwiegend im Kölner Raum - anzutreffen. Fünf dieser zehn VHS-Webseiten weisen mindestens ein solches Angebot aus - wie beispielweise

- „Brücken bauen: Mülheimer Stadtteilmütter“ - ein Projekt der VHS Köln und in Trägerschaft der Christlichen Sozialhilfe Köln e. V. zur Qualifizierung von Frauen mit Migrationshintergrund zu Stadtteilmüttern.

\footnotetext{
8 In der Online-Befragung wurde die Kategorie des Migrationshintergrunds mit Verweis auf die Definition des Statistischen Bundesamtes eingeführt.

9 Nicht unerwähnt bleiben sollen in diesem Zusammenhang auch die interkulturellen Weiterbildungsangebote für ehrenamtliche und hauptamtliche Fachkräfte (wie z. B. Fortbildungen zu kultureller Vielfalt und zum Umgang mit Kulturen für Dozentinnen und Dozenten und Referentinnen und Referenten, Anti-Rassismus-Trainings für Lehrende und Sozialarbeiterinnen und Sozialarbeiter und zur Kultursensibilität und Qualifizierung von Ehrenamtlichen) (vgl. Öztürk und Reiter 2017, S. 96 ff.).
} 


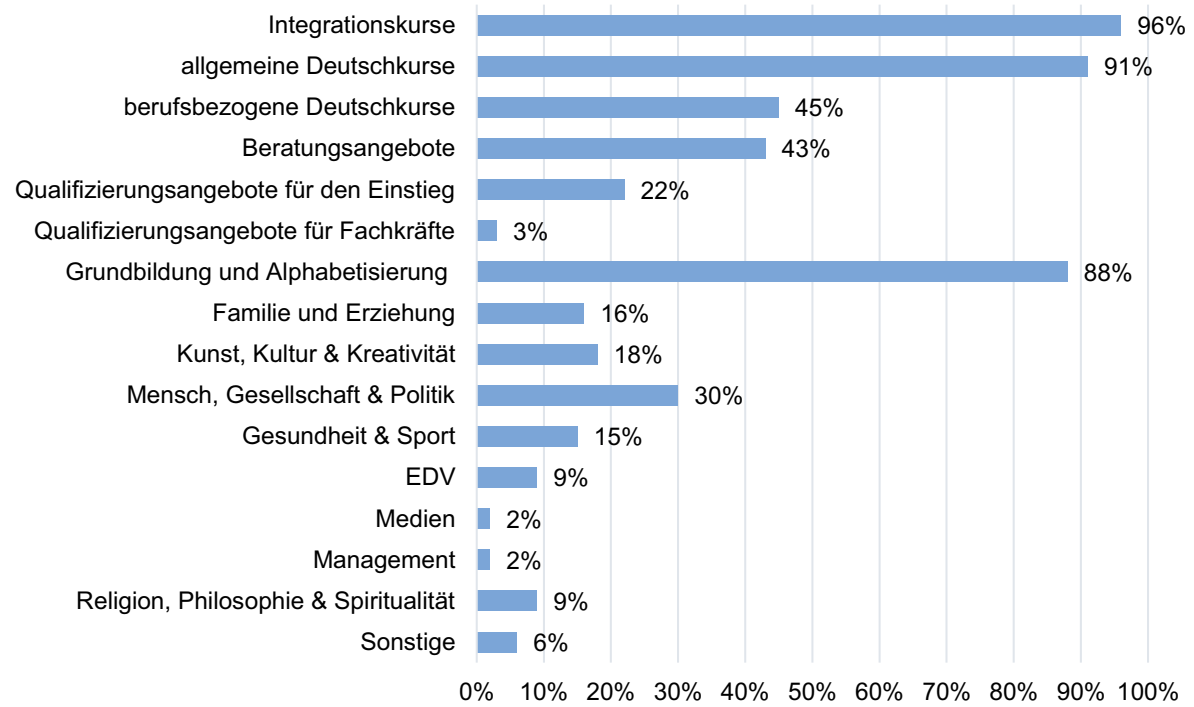

Abb. 1 Zielgruppenangebote für Erwachsene mit Migrationshintergrund nach Themenfeldern. Angaben in Prozent, Mehrfachnennungen möglich; $n=67$

- „WelcomeWalk - Freiwillige und Geflüchtete erkunden gemeinsam die Stadt“ein Workshop der VHS Köln in Zusammenarbeit mit der Kölner Freiwilligen Agentur, in dessen Rahmen im Tandem-Verfahren jeweils eine Ortskundige bzw. ein Ortkundiger und eine Neu-Kölnerin bzw. ein Neu-Kölner in einem Spaziergang die Stadt Köln erkunden.

- „Internationales Bildungszentrum - Die Brücke“ - ein Angebot der VHS Düsseldorf in Kooperation mit 30 Auslandsgesellschaften bzw. Migrantenorganisationen insbesondere für den interkulturellen Austausch.

Auf VHS-Webseiten in Landkreisen mit einem niedrigen Bevölkerungsanteil an Personen mit Migrationshintergrund konnten über den engen zielgruppenspezifischen Rahmen hinausgehende, partizipative bzw. dialogorientierte Angebote nicht identifiziert werden.

In der Zusammenschau von zielgruppenspezifischen Angeboten kann festgehalten werden, dass die Bevölkerungsgruppe mit Migrations- und Fluchthintergrund bereits auf vielfältige Weise adressiert wird. Der Schwerpunkt dieser Angebote wird auch hierin deutlich: Es häufen sich Klagen über fehlende Kursplätze insbesondere in Deutsch- und Integrationskursen und das nicht erst aufgrund der starken Fluchtzuwanderung 2015 (s. z. B. Bilger und Hartmann 2011, S. 100), die in diesem Zusammenhang lediglich verstärkend wirkte. Infolgedessen sind die Kapazitäten vieler Anbieter von Deutsch- und Integrationskursen in Deutschland bereits ausgeschöpft. In vielen Einrichtungen bestehen lange Wartelisten. ${ }^{10}$ Die entsprechenden Landes-

10 Siehe beispielsweise Adler (2015), DVV International (2016) und Hübert (2015). 


\section{Der Teilnehmeranteil am Regelangebot...}

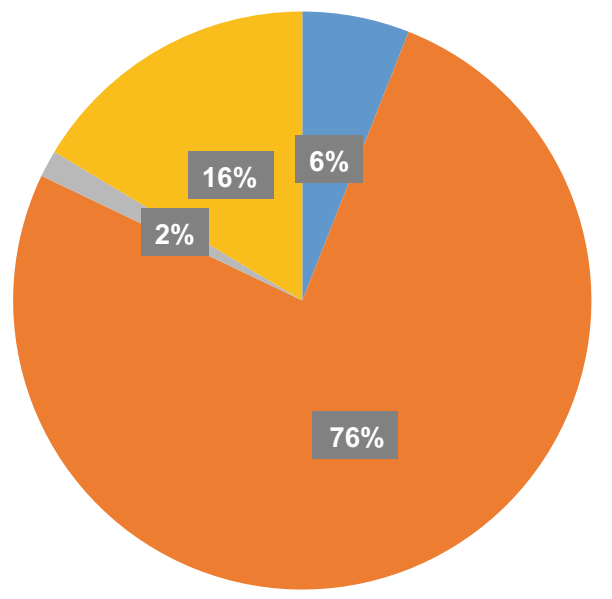

\author{
-...entspricht dem Anteil der \\ Bevölkerung mit Migrationshintergrund \\ im Bezirk unserer Einrichtung. \\ -...ist geringer als der Anteil der \\ Bevölkerung mit Migrationshintergrund \\ im Bezirk unserer Einrichtung. \\ -...ist höher als der Anteil der \\ Bevölkerung mit Migrationshintergrund \\ im Bezirk unserer Einrichtung.
}

Das kann ich nicht einschätzen.

Abb. 2 Teilnahme von Personen mit Migrationshintergrund am Regelangebot in der Einschätzung der befragten VHS in Prozent; $n=67$

verbände der VHS ${ }^{11}$ und insbesondere der DVV machen u. a. darauf aufmerksam, dass die VHS die „Nachfrage nach Bildungs- und Integrationsangeboten nicht mehr bewältigen“ können und fordert die „Förderung von Bund und Ländern zum Ausbau von Angeboten und Strukturen“ zur Stärkung der VHS ,als kommunale Zentren für Integration“ (DVV 2015a, S. 1).

Neben zielgruppenspezifischen Kursangeboten bietet die VHS entsprechend ihrem gesetzlich verankerten Bildungsauftrag auch eine Vielzahl von Kursen, Vorträgen, Workshops, Exkursionen und Studienfahrten zu verschiedensten Themen, die sich nicht nur an eine bestimmte Zielgruppe richten (das sog. Regelangebot). Wie es um den Teilnahmeanteil von Personen mit Migrationshintergrund konkret am Regelangebot bestellt ist, lässt sich nur bedingt beantworten, zumal solche breit angelegten Datenquellen bislang fehlen (vgl. Öztürk 2014, S. 53). Vor diesem Hintergrund wurden die VHS um eine Einschätzung des Teilnahmeanteils von Personen mit Migrationshintergrund an solchen offenen Angeboten gebeten. Die befragten VHS geben dazu folgende Einschätzung ab (s. Abb. 2):

Eine überwiegende Mehrheit der befragten VHS schätzte den Anteil von Teilnehmenden mit Migrationshintergrund am Regelangebot geringer als den Anteil der Bevölkerung mit Migrationshintergrund im jeweiligen Einzugsgebiet (76,1\%). 4 von 67 VHS, also rund sechs Prozent, sagen, dass der Anteil entsprechend dem des Bevölkerungsanteils mit Migrationshintergrund ist und lediglich eine einzige VHS $(1,5 \%)$ schätzte den Anteil höher ein. Auffallend ist hier allerdings auch, dass 11 von 67 VHS (16,4\%) diese Einschätzung nicht vornehmen können. Hinzu kommt noch, dass hier vier VHS keine Angabe machten.

\footnotetext{
${ }^{11}$ Siehe beispielsweise Gesprächskreis für Landesorganisationen der Weiterbildung in NRW (2016) und Landesverband der Volkshochschulen von NRW (2015).
} 
Diese Einschätzungen ${ }^{12}$ zeigen auch Parallelen zum wiederkehrenden Befund einer verhältnismäßig niedrigen Beteiligung insbesondere von Personen der ersten Zuwanderergeneration an Weiterbildung auf. Diese wird häufig auf individuelle Teilhabebarrieren wie geringe Deutschsprachkenntnisse, ungünstige Positionierung im Erwerbsleben, mangelnde Information über Weiterbildungsmöglichkeiten und familiäre Verpflichtungen zurückgeführt (vgl. Kuwan und Seidel 2013, S. 221; Öztürk und Reiter 2016, S. 53 ff.). Eher unberücksichtigt bleiben dabei organisationsbezogene Barrieren, die einen nicht unwesentlichen Einfluss auf die Teilhabe an Weiterbildung haben. Umso wichtiger erscheinen daher die Bemühungen der VHS um eine nachhaltige Verbesserung der Weiterbildungsbeteiligung von Personen mit Migrations- und Fluchthintergrund. Insofern ist es konsequent, dass es nahezu allen befragten VHS in NRW $(92,4 \%)$ ein dringendes Anliegen ist, zukünftig mehr Teilnehmende mit Migrationshintergrund zu erreichen und damit eine gleichberechtigte Weiterbildungsteilhabe zu fördern. Entsprechend dieser Zielsetzung nutzen die VHS bereits Kooperationen mit anderen Einrichtungen und Netzwerken (u. a. Stadtverwaltungen, Wohlfahrtsverbände, Migrantenselbstorganisationen), setzen mehrsprachige Materialien und Lehrende ein, oder konzipieren niedrigschwellig aufsuchende Angebote (vgl. Öztürk und Reiter 2017, S. 91 ff.).

\subsection{Umgang auf Ebene des Personals}

Auf Ebene des Personals stellen sich Fragen nach der Beschäftigungssituation von, der Erwartungshaltung gegenüber sowie der Nutzung spezifischer Strategien zur Rekrutierung von Personen mit Migrationshintergrund.

Die große Mehrheit der VHS in NRW (70,8\%) beschäftigt Personen mit Migrationshintergrund. Diese arbeiten meistens als Honorarkraft $(95,7 \%)$, mit deutlichem Abstand folgen Positionen in der Verwaltung bzw. im Sekretariat $(39,1 \%)$ oder als pädagogische Mitarbeitende (34,8\%). Nur ein kleiner Teil der VHS (8,7\%) hat angegeben, Personen mit Migrationshintergrund als Angestellte in Leitungsfunktion zu beschäftigen (vgl. Öztürk und Reiter 2017, S. 72 f.). Dieses Ergebnis überrascht nicht völlig, da beispielsweise der Anteil der freien bzw. nebenberuflichen Mitarbeiter im gesamten Weiterbildungsbereich traditionell überdurchschnittlich hoch ist (vgl. Martin et al. 2016).

Unabhängig hiervon wird der zukünftige Bedarf an Mitarbeitenden mit Migrationshintergrund in VHS relativ hoch eingeschätzt, wenngleich ebenfalls eher im Bereich der freien bzw. pädagogischen Mitarbeitenden und weniger im Bereich der Mitarbeitenden in Leitungsfunktion. Dieser Bedarf mündet jedoch selten explizit in spezifische Rekrutierungsstrategien, die auf eine gezielte Anwerbung von Personen mit Migrationshintergrund abstellen. Lediglich bei einem kleinen Teil der VHS werden gezielte Strategien zur Rekrutierung von Mitarbeitenden mit Migrationshin-

\footnotetext{
12 Aufgrund des Online-Charakters der Befragung haben die Autorinnen und Autoren der Studie keinen Einblick in das genaue Vorgehen bei der Einschätzung. Da sich hier einige VHS skeptisch gegenüber entsprechenden Erhebungen in ihren Teilnehmerstatistiken äußerten, da sie sie als stigmatisierend einschätzen, kann angenommen werden, dass einige der befragten VHS ihre Einschätzung ohne Datengrundlage gemacht haben.
} 
tergrund eingesetzt, während der Großteil (74,2\%) keine Rekrutierungsstrategien benennt.

Diejenigen VHS, die Personen mit Migrationshintergrund gezielt ansprechen, nannten am häufigsten die Rekrutierungsstrategie der „Benennung von Diversity bzw. Interkultureller Kompetenz als Einstellungsvoraussetzung“ in den Stellenprofilen (19,4\%), es folgen die „Hervorhebung, dass Bewerbungen von Menschen mit Migrationshintergrund besonders berücksichtigt werden“ (6,5\%) und die ,Veröffentlichung in Medien, in denen Menschen mit Migrationshintergrund erreicht werden“" $(1,6 \%)$. Unter ,,andere Strategien“ wurden ,,persönliche Kontakte“ und die Nutzung von Netzwerken und Kooperationen genannt.

Die VHS, die keine Mitarbeitenden mit Migrationshintergrund beschäftigen, sehen die Gründe dafür vor allem in fehlenden Bewerbungen von Personen mit Migrationshintergrund und auch darin, dass die Ressourcen weitere Stellenbesetzungen nicht zulassen.

\subsection{Umgang auf Ebene der Organisation}

Zentrale Aspekte auf dieser Analyseebene sind die Verankerung der Anerkennung von Vielfalt im Leitbild, sowie Erfahrungen mit organisationsbezogenen Konzepten wie Diversity Management und Interkultureller Öffnung.

Mehr als jede zweite der befragten VHS (57,8\%) hat bereits die Anerkennung (migrationsbedingter) Vielfalt im Leitbild verankert; der durch den wbmonitor ermittelte bundesweite Durchschnitt liegt bei 64\% (vgl. Ambos et al. 2017, S. 34). Die größte VHS in NRW, die Volkshochschule Köln, bringt dies beispielweise in ihrem Leitbild wie folgt zum Ausdruck:

„Wir stehen für eine gleichberechtigte, demokratische Gesellschaft, für ein dauerhaft friedliches Zusammenleben von Menschen verschiedener Generationen, Geschlechter, Nationalitäten, Kulturen sowie Weltanschauungen und Religionen. Wir begreifen die Vielfalt der Kölner Stadtgesellschaft als Stärke und leisten selbst einen Beitrag zur Integration und Inklusion. Für uns ist es normal, verschieden zu sein“ (https://vhs-koeln.de/Artikel/cmx548825f746ebd.html).

Die VHS Köln steht damit im Einklang mit dem bereits am 2. März 2011 auf der Mitgliederversammlung des DVV verabschiedeten Beschluss, die VHS ,weiter zu interkulturellen Weiterbildungs- und Begegnungszentren“ $\mathrm{zu}^{\mathrm{a}}$ entwickeln und künftig noch mehr in diesem Bereich tätig zu werden, z. B. durch den Ausbau von Angeboten ,für die soziale, kulturelle und wirtschaftliche Integration von Menschen mit Migrationshintergrund“ (DVV 2011, S. 7).

In Bezug auf die Konzepte Interkultureller Öffnung und Diversity Management geben mehr als zwei Drittel $(70,4 \%)$ der befragten VHS an, mindestens eines der beiden organisationsbezogenen Konzepte zu kennen, wobei Interkulturelle Öffnung $(26,2 \%)$ bekannter ist als Diversity Management (13,1\%) (vgl. Öztürk und Reiter 2017, S. 64). Dieses Ergebnis ist erwartungsgemäß, da das Konzept der Interkulturellen Öffnung gerade in vielen großstädtischen VHS besonders präsent ist (vgl. DVV 2015b). Es dürfte künftig noch stärkere Beachtung finden, zumal die VHS ,sich auf ihre Fahnen - sprich in ihr gemeinsames Arbeitsprogramm - geschrie- 
ben [haben], dass sie in den kommenden Jahren die interkulturelle Öffnung weiter forcieren wollen, weil auf kommunaler Ebene ein Ort der Integration und des interkulturellen Miteinanders dringend benötigt wird“, so Ernst Dieter Rossmann und Ulrich Aengenvoort vom DVV (2016, S. 4). Immerhin fast ein Drittel der befragten VHS $(29,5 \%)$ kennt jedoch weder das Konzept Interkulturelle Öffnung noch das des Diversity Managements (vgl. Öztürk und Reiter 2017, S. 64).

Wenngleich der Bekanntheitsgrad der genannten Konzepte insgesamt sehr hoch ist, weisen rund zwei Drittel der VHS (62,5\%) keinerlei Erfahrungen mit ihrer Umsetzung auf. Falls Umsetzungserfahrungen vorliegen, dann mit Interkultureller Öffnung (17,5\%). Auf Erfahrungen mit Diversity Management bzw. mit beiden Konzepten können jeweils zehn Prozent der VHS zurückgreifen (vgl. ebd.). In VHS, die eines der oder beide Konzepte bereits umsetzen, existieren jedoch lediglich punktuelle Einzelmaßnahmen. Ein Beispiel hierfür ist die Formulierung eines entsprechenden Leitbilds, der jedoch keine weiteren Maßnahmen etwa auf Ebene des Personals oder der Angebote folgen.

\section{Fazit und Herausforderungen}

Im Fokus dieses Beitrags standen speziell die VHS und deren Umgang mit Migration und Flucht auf der Ebene der Angebote, sowie des Personals und der Organisation. Die vorgestellten, ausgewählten Ergebnisse zeigen das deutlich gewachsene Interesse der VHS am organisationsweiten Umgang mit migrations- und fluchtbedingter Diversität. Die Bemühungen der VHS in dieser Hinsicht sind durchaus zahlreich und vielfältig, allerdings unterschiedlich weit vorangeschritten.

Der weit überwiegende Teil der VHS hat Kenntnis von Konzepten, die auf eine wirksame Veränderung der gesamten Organisationskultur zielen. Allerdings liegen hier noch wenig konkrete Anwendungserfahrungen vor. Viele VHS haben jedoch bereits entsprechende Gestaltungsmaßnahmen eingeleitet, wobei großstädtische VHS hier weiter vorangeschritten sind als (klein-)städtische und ländliche VHS. Wenngleich die Einbettung entsprechender Gestaltungsmaßnahmen in eine kohärente Gesamtstrategie noch fehlt, wurde die Notwendigkeit von Veränderungen auf organisationaler Ebene entsprechend des sozialen und gesellschaftlichen Wandels erkannt.

Die vielfältigen beharrlichen Bemühungen vieler VHS um eine gerechte und stärkere Teilhabe von Menschen mit Migrationshintergrund an Weiterbildung zeigen sich jedoch am deutlichsten auf der Ebene der Angebote. Nahezu alle VHS haben bereits spezielle Angebote für Personen mit Migrationshintergrund im Programm und nehmen dabei vor allem Bezug auf die Umsetzung integrationspolitischer Maßnahmen, aber auch auf die Förderung der Grundbildung von Erwachsenen mit Migrationshintergrund. Unklar bleibt dabei, wie es mit der Repräsentanz von Menschen mit Migrationshintergrund im Regelangebot der VHS konkret bestellt ist. Diese wird ganz überwiegend als gering eingeschätzt. Es konnten jedoch auch einige Beispiele für partizipative bzw. dialogorientierte Angebote sowie Angebote nicht nur für, sondern auch mit Menschen mit Migrationshintergrund oder geflüchteten Erwachsenen aufgezeigt werden. 
Insgesamt betrachtet zeigen die befragten VHS im Umgang mit Migration und Flucht auf Ebene der Angebote, des Personals und der Organisation großes Interesse und Engagement. Die Befunde verdeutlichen aber auch, dass bei der Entwicklung und Umsetzung wirkungsvoller organisationsumfassender Strategien und Maßnahmen noch Handlungsbedarf besteht, vor allem in den folgenden Bereichen (s. hierzu ausführlich Öztürk und Reiter 2017, S. 106 ff.):

- Organisationsentwicklung: VHS sind gefordert, ihre institutionalisierten Handlungspraxen unter Rückgriff auf Organisationsentwicklungsansätze wie Interkulturelle Öffnung und/oder Diversity Management beharrlich zu reflektieren und diversitätsbewusst weiterzuentwickeln, organisationsbedingte Zugangsbarrieren zu beseitigen sowie insgesamt das organisationale Lernen (s. z. B. Argyris und Schön 2006; Schein 2010) durchgreifend zu fördern.

- Personalentwicklung: VHS sind gefordert, ihr gesamtes Personal (Leitung, Lehrende, Verwaltung) stärker für die Belange der (potenziellen) Teilnehmenden ob mit oder ohne Migrationshintergrund - und insgesamt für die gesellschaftliche Vielfalt - zu sensibilisieren. Insofern sollten alle Mitarbeitenden über mögliche Schritte zur Durchführungen von Veränderungen etwa im Kontext von Interkultureller Öffnung bzw. Diversity Management nicht nur informiert bzw. mit anliegenden Aufgaben versehen werden, sondern mitbestimmend beteiligt werden. Hinsichtlich der Rekrutierung neuen Personals sollten Potenziale einer gezielten Ansprache genutzt und generell ein ,transparentes“ Bewerbungsverfahren angestrebt werden.

- Angebotsentwicklung: VHS sind gefordert, ihre langjährigen Erfahrungen mit zielgruppenspezifischen Kursen für Menschen mit Migrationshintergrund verstärkt in der Gestaltung von Übergängen zwischen Zielgruppenangeboten, wie etwa den Deutsch- und Integrationskursen, und dem Regelangebot zu nutzen (vgl. Lücker und Mania 2014; Zimmer et al. 2015). Ausgewählte Deutsch- und Integrationskurse in VHS sollten in der Übergangsberatung in das Regelangebot eine wichtige Funktion übernehmen. Im Rahmen des Kursunterrichts könnten beispielsweise der konkret bestehende Bildungsbedarf und die individuellen Bildungsinteressen systematisch ermittelt ${ }^{13}$ und gezielte Informationen über Angebote und Aktivitäten sowie Fördermöglichkeiten weitergegeben werden (vgl. Öztürk 2016, S. 329 ff.). Vordringlich ist deshalb die Aufwertung solcher Angebote durch bessere finanzielle und personelle Rahmenbedingungen. Denn neben bewusst ,integrativ“ gestalteten Angeboten bietet vor allem das Regelangebot den Raum, plurale Überzeugungen kennen und respektieren zu lernen. Die gesellschaftliche Vielfalt auch hier widerzuspiegeln, ist damit nicht nur im Interesse bislang unterrepräsentierter Teilnehmendengruppen, sondern auch aller Teilnehmenden und Akteurinnen und Akteure der Weiterbildung.

13 Hierzu gibt es mittlerweile eine breite Auswahl an Methoden und Instrumenten zur Ermittlung des Weiterbildungsbedarfs (s. z. B. Kauffeld 2016, S. 23 ff.; Wegerich 2015, S. 97 ff.), die sich (ggfs. nach Modifikation) einsetzen lassen. 
Schließlich ist besonders zu unterstreichen, dass der DVV als Dachverband der VHS in Deutschland in seinem Positionspapier bereits die Notwendigkeiten formuliert hat, stärker auf die Zielgruppe Erwachsener mit Migrationshintergrund Bezug zu nehmen und dafür auch die gesamte Organisation weiterzuentwickeln (vgl. DVV 2015a, S. 3).

All diese Anforderungen können zwar ohne zusätzliche finanzielle und personelle Ausstattung kaum bewerkstelligt werden, aber die VHS können hier weiterhin eine richtungsweisende Rolle einnehmen und noch sichtbarer ein Zeichen für eine diversitätsorientierte Organisations- und Personalentwicklung sowie für eine adressatengerechte Programm- und Angebotsplanung setzen. Der Weiterbildungsforschung kommt hier die vordringliche Aufgabe zu, konsequent sowohl Programm- und Angebotsstrukturen als auch diversitätsorientierte Organisations- und Personalentwicklungsstrategien stärker in den Blick zu nehmen und dabei verwendete Konzepte empirisch zu prüfen und theoretisch zu reflektieren.

Open Access Dieser Artikel wird unter der Creative Commons Namensnennung 4.0 International Lizenz (http://creativecommons.org/licenses/by/4.0/deed.de) veröffentlicht, welche die Nutzung, Vervielfältigung, Bearbeitung, Verbreitung und Wiedergabe in jeglichem Medium und Format erlaubt, sofern Sie den/die ursprünglichen Autor(en) und die Quelle ordnungsgemäß nennen, einen Link zur Creative Commons Lizenz beifügen und angeben, ob Änderungen vorgenommen wurden.

\section{Literatur}

Adler, E. (2015). Deutschkurse an Volkshochschulen sind Mangelware. WAZ vom 26.08.2015. https://www. waz.de/politik/deutschkurse-an-volkshochschulen-sind-mangelware-id11021180.html. Zugegriffen: 14. Jan. 2018.

Ambos, I., Koscheck, S., \& Martin, A. (2017). Kulturelle Vielfalt. Ergebnisse der wbmonitor Umfrage 2016. https://www.bibb.de/veroeffentlichungen/de/publication/download/8325. Zugegriffen: 12. Febr. 2018.

Argyris, C., \& Schön, D. A. (2006). Die lernende Organisation: Grundlagen, Methode, Praxis (3. Aufl.). Stuttgart: Klett-Cotta.

Bilger, F., \& Hartmann, J. (2011). Weiterbildung von Personen mit Migrationshintergrund. In F. Bilger \& B. von Rosenbladt (Hrsg.), Weiterbildungsbeteiligung 2010. Trends und Analysen auf Basis des deutschen AES (S. 97-102). Bielefeld: W. Bertelsmann.

BMBF - Bundesministerium für Bildung und Forschung (2018). Weiterbildungsverhalten von Personen mit Migrationshintergrund. Ergebnisse der erweiterten Erhebung des Adult Education Survey (AES-Migra 2016). Bielefeld. https://www.bmbf.de/pub/Weiterbildungsverhalten_in_Deutschland_ 2016.pdf. Zugegriffen: 22. Jul. 2018.

DVV International - Institut für Internationale Zusammenarbeit des Deutschen Volkshochschul-Verbandes e. V. (2016). Menschen auf der Flucht: Eine Herausforderung für die Erwachsenenbildung. Bonn. https://www.dvv-vhs.de/fileadmin/user_upload/1_Startseite/IPE76_DE_web.pdf. Zugegriffen: 18. Jan. 2018

DVV - Deutscher Volkshochschul-Verband e. V. (2011). Die Volkshochschule - Bildung in öffentlicher Verantwortung. Bonn: DVV.

DVV - Deutscher Volkshochschul-Verband e.V. (2015a). Bildungsoffensive für Flüchtlinge. Bonn. https://www.dvv-vhs.de/fileadmin/user_upload/1_Startseite/Positionspapier_Bildungsoffensive_ fuer_Fluechtlinge_2_2_.pdf. Zugegriffen: 12. Jan. 2018.

DVV - Deutscher Volkshochschul-Verband e. V. (2015b). Positionierung der Volkshochschulen in der Einwanderungsgesellschaft. Bonn. https://www.dvv-vhs.de/fileadmin/user_upload/6_Themenfelder/ Integration/Dokumente/Positionierung_der_Volkshochschulen_in_der_Einwanderungsgesellschaft_ 23072015.pdf. Zugegriffen: 12. Jan. 2018. 
DVV - Deutscher Volkshochschul-Verband e. V. (2016). Jahresbericht 2015/2016. Bonn. https://www. dvv-vhs.de/fileadmin/user_upload/3_Der_Verband/Presse/Jahresberichte/DVV-Jahresbericht-20152016_Webversion.pdf. Zugegriffen: 20. Jan. 2018.

von Eschenbach, M.E. (2016). „Was ist Migration?“ Risiken eines essentialistischen Migrationsbegriffs in der Erwachsenenbildung. Zeitschrift für Weiterbildungsforschung, 39, 43-59.

Europäische Union (2015). Amtsblatt der Europäischen Union Nr. C417 vom 15.12.2015. Neue Prioritäten für die europäische Zusammenarbeit auf dem Gebiet der allgemeinen und beruflichen Bildung. http://eur-lex.europa.eu/legal-content/DE/TXT/PDF/?uri=CELEX:52015XG1215(02)\&from=DE. Zugegriffen: 12. Jan. 2018.

Falkenstern, A., \& Käpplinger, B. (2017). Flucht und Migration in der Erwachsenenpädagogik - Zeithistorische Vergleiche. In Die Dekanin Fachbereich 03 Sozial- Kulturwissenschaften der JustusLiebig-Universität (Hrsg.), Jahrbuch 2017. Schwerpunkt Flucht und Migration (S. 12-13). Gießen: Justus-Liebig-Universität. https://www.uni-giessen.de/fbz/fb03/dek1/Info/d1/FB03_Jahrbuch2017. pdf. Zugegriffen: 12. Jul. 2018.

Gesprächskreis für Landesorganisationen der Weiterbildung in NRW (2016). Positionspapier der gemeinwohlorientierten Weiterbildung zum geplanten Integrationsplan für NRW. Düsseldorf. http:// www.vhs-nrw.de/fileadmin/user_upload/3_Positionspapier_Weiterbildung_NRW_07.03.2016_mit_ Logos.pdf. Zugegriffen: 12. Jan. 2018.

Gnahs, D., \& Bilger, F. (2013). Anbieter auf dem Markt der Weiterbildung. In F. Bilger, D. Gnahs, J. Hartmann \& H. Kuper (Hrsg.), Weiterbildungsverhalten in Deutschland. Resultate des Adult Education Survey 2012 (S. 110-124). Bielefeld: W. Bertelsmann.

Heinemann, A.M.B. (2018). Institutionelle Öffnung und Migrationsgesellschaft - einige rahmende Anmerkungen. In A. M. B. Heinemann, M. Stoffels \& S. Wachter (Hrsg.), Erwachsenenbildung für die Migrationsgesellschaft. Institutionelle Öffnung als diskriminierungskritische Organisationsentwicklung (S. 11-39). Bielefeld: W. Bertelsmann.

Hübert, H. (2015). Wartelisten für Integrationskurse. In Deutschlandfunk vom 5.10.2015. http://www. deutschlandfunk.de/fluechtlingsansturm-wartelisten-fuer-integrationskurse.680.de.html?dram:article id=333053. Zugegriffen: 12. Jan. 2018.

Huntemann, H., \& Reichart, E. (2015). Volkshochschul-Statistik - 53. Folge, Arbeitsjahr 2014, Bonn. https://www.die-bonn.de/doks/2015-volkshochschule-statistik-36.pdf. Zugegriffen: 12. Jan. 2018.

Huntemann, H., \& Reichart, E. (2017). Volkshochschul-Statistik - 55. Folge, Arbeitsjahr 2016, Bonn. https://www.die-bonn.de/doks/2017-volkshochschule-01.pdf. Zugegriffen: 12. Jan. 2018.

Kauffeld, S. (2016). Nachhaltige Personalentwicklung und Weiterbildung. Betriebliche Seminare und Trainings entwickeln, Erfolge messen, Transfer sichern (2. Aufl.). Berlin \& Heidelberg: Springer.

Kuwan, H., \& Seidel, S. (2013). Weiterbildungstransparenz und Weiterbildungsberatung. In F. Bilger, D. Gnahs, J. Hartmann \& H. Kuper (Hrsg.), Weiterbildungsverhalten in Deutschland. Resultate des Adult Education Survey 2012 (S. 232-247). Bielefeld: W. Bertelsmann.

Landesverband der Volkshochschulen NRW e.V. (2015). Resolution der Mitgliederversammlung des Landesverbandes der Volkshochschulen von NRW e.V. - Flüchtlinge brauchen Sprache. Bielefeld. http:// www.vhs-nrw.de/uploads/media/20150522_Resolution_MV_08.05.2015_Fluechtlinge_fdh_01.pdf. Zugegriffen: 10. Jan. 2018.

Lücker, L., \& Mania, E. (2014). Übergänge innerhalb der Weiterbildungseinrichtungen am Beispiel von Angeboten für Menschen mit Migrationshintergrund. Report - Zeitschrift für Weiterbildungsforschung, 2, 71-83.

MAIS - Ministerium für Arbeit, Integration und Soziales des Landes Nordrhein-Westfalen (2016). Integrationsprofile der kreisfreien Städte und Kreise in NRW. Ausgabe 2016. Düsseldorf. http://www. integrationsmonitoring.nrw.de/integrationsberichterstattung_nrw/Integration_kommunal/Integrations profile/Ausgabe_2016/index.php. Zugegriffen: 10. Jul. 2018.

Martin, A., Lencer, S., Schrader, J., Koscheck, S., Ohly, H., Dobischat, R., Elias, A., \& Rosendahl, A. (2016). Das Personal in der Weiterbildung. Arbeits- und Beschäftigungsbedingungen, Qualifikationen, Einstellungen zu Arbeit und Beruf. Bielefeld: wbv.

Mayring, P. (2015). Qualitative Inhaltsanalyse. Grundlagen und Techniken (12. Aufl.). Weinheim: Beltz. Olbrich, J. (2001). Geschichte der Erwachsenenbildung in Deutschland. Opladen: Leske + Budrich.

Öztürk, H. (2014). Migration und Erwachsenenbildung. Bielefeld: W. Bertelsmann.

Öztürk, H. (2016). Diversitätsbewusste Weiterbildung im Migrationskontext. Anforderungen an das Zusammenspiel von Weiterbildungsforschung, -praxis und -politik. Hessische Blätter für Volksbildung, 4, 324-333. 
Öztürk, H., \& Kaufmann, K. (2009). Migration background and participation in continuing education in Germany: an empirical analysis based on data from the German Socio-Economic Panel study (SOEP). European Educational Research Journal, 8, 255-275.

Öztürk, H., \& Reiter, S. (2016). Berufliche Weiterbildungsteilhabe von Erwachsenen mit „Migrationshintergrund“ unter Berücksichtigung ausgewählter Diversitätsaspekte anhand des Nationalen Bildungspanels. In K. Dollhausen \& S. Muders (Hrsg.), Diversität und lebenslanges Lernen. Aufgaben für die organisierte Weiterbildung (S. 47-64). Bielefeld: W. Bertelsmann.

Öztürk, H., \& Reiter, S. (2017). Migration und Diversität in Einrichtungen der Weiterbildung. Bielefeld: W. Bertelsmann.

Robak, S., \& Petter, I. (2014). Programmanalyse zur interkulturellen Bildung in Niedersachsen. Bielefeld: wbv. www.die-bonn.de/doks/2014-interkulturelle-bildung-01.pdf. Zugegriffen: 24. Jul. 2018

Ruhlandt, M. (2016). Erwachsenenbildung in der Einwanderungsgesellschaft. Organisationale Voraussetzungen Interkultureller Offenheit in Einrichtungen der Weiterbildung. Wiesbaden: Springer.

Schein, E. H. (2010). Organisationskultur (3. Aufl.). Bergisch Gladbach: EHP-Verlag Andreas Kohlhage.

Statistisches Bundesamt (2017). Bevölkerung und Erwerbstätigkeit. Bevölkerung mit Migrationshintergrund - Ergebnisse des Mikrozensus 2016. https://www.destatis.de/DE/Publikationen/Thematisch/ Bevoelkerung/MigrationIntegration/Migrationshintergrund2010220167004.pdf. Zugegriffen: 24. Jul. 2018.

Süssmuth, R., \& Eisfeld, K.H. (2018). Volkshochschule. Erwachsenenbildung/Weiterbildung in öffentlicher Verantwortung. In R. Tippelt \& A. von Hippel (Hrsg.), Handbuch Erwachsenenbildung/ Weiterbildung (6. Aufl., S. 763-784). Wiesbaden: Springer VS.

Vanderheiden, E., \& Mayer, C.-H. (Hrsg.). (2014). Handbuch Interkulturelle Öffnung. Grundlagen, Best Practice, Tools. Göttingen: Vandenboeck \& Ruprecht.

Walgenbach, K. (2017). Heterogenität - Intersektionalität - Diversity in der Erziehungswissenschaft (2. Aufl.). Opladen und Toronto: Budrich.

Wegerich, C. (2015). Strategische Personalentwicklung in der Praxis. Instrumente, Erfolgsmodelle, Checklisten, Praxisbeispiele (3. Aufl.). Berlin, Heidelberg: Springer.

Zimmer, V., Fleige, M., \& Thom, S. (2015). Zugang von Menschen mit Migrationshintergrund zu Weiterbildungsangeboten. Bonn. https://www.die-bonn.de/doks/interkulturelle-bildung-02.pdf. Zugegriffen: 11. Jul. 2018. 